\title{
Electrical characterization of aeronautical nanocomposites supported by Tunneling AFM (TUNA)
}

\author{
Patrizia Lamberti ${ }^{1 *}$, Giuseppina Barra $^{2}$, Liberata Guadagno $^{2}$, Khalid Lafdi $^{3}$, Carlo \\ Naddeo $^{2}$, Marialuigia Raimondo ${ }^{2}$, Giovanni Spinelli ${ }^{1}$, Vincenzo Tucci ${ }^{1}$ and Luigi \\ Vertuccio $^{2}$ \\ ${ }^{1}$ Department of Information and Electrical Engineering and Applied Mathematics, University of \\ Salerno, Via Giovanni Paolo II, 132, 84084, Fisciano (SA), Italy \\ ${ }^{2}$ Department of Industrial Engineering, University of Salerno, Via Giovanni Paolo II, 132, 84084, \\ Fisciano (SA), Italy \\ ${ }^{3}$ University of Dayton, 300 College Park, Dayton Ohio, 45440, USA
}

\begin{abstract}
Epoxy nanocomposites fulfill tight and compelling industrial requirements in the field of structural material for aeronautical applications. In this paper the development and characterization of nanocomposites obtained by filling tetrafunctional epoxy resin (tetraglycidyl methylene dianiline cured with the aromatic diamine 4,4'-diaminodiphenylsulfone, named T20BD) with carbon nanofibers (CNF) is discussed. A filler amount ranging from $0.05 \%$ to $2 \% \mathrm{wt}$ is considered. The DC volume conductivity and the dielectric characteristics $\left(\epsilon^{\prime}\right)$ of the nanocomposites in the frequency range $100 \mathrm{~Hz}-1 \mathrm{MHz}$ are analyzed and compared with those of the pure resin. Atomic force microscopy, mapping the local topography by means of tunneling effect, is used for recording the electrical percolation path for nanocomposites. In particular, the case $1.3 \mathrm{wt} \%$ of $\mathrm{CNF}$ filled nanocomposites that exhibits a stable behavior of the conductivity in the full investigated frequency range, is here reported. The developed filled epoxy used in carbon fiber reinforced composites, shows enhanced electrical properties leading to better electromagnetic (EM) performances in EM coatings, EM shields and filters or radar absorber materials (RAMs).
\end{abstract}

\section{Introduction}

Since their discovery, due to their unique physical, chemical and electrical properties, carbon nanotubes (CNTs) and others related one-dimensional filler such as carbon nanofibers (CNFs) opened a wide range of applications in many fields of science and engineering allowing to obtain new materials and new electronic devices. In the last decade many research efforts have been devoted toward studies on polymer nanocomposites filled with such highly conductive additives given their peculiar and enhanced properties with respect to those of the neat resin. These improvements strongly depend on the chemistry of polymer matrices, nature

\footnotetext{
* Corresponding author: plamberti@unisa.it
} 
of nanofillers and their mutual interactions $[1,2,3]$. In particular, the improvements are originated by the huge filler/polymer matrix interface which allows to transfer the notable properties of the fillers to the resulting composites. This in turn has a major influence on the electrical, thermal and mechanical properties of the resulting nanocomposite systems [4]. In particular, the introduction of very small weight percentages of high aspect ratio (the ratio between length and diameter) carbon nanostructures leads to a sharp insulation-conduction transition in the resulting polymer-based composite due to the formation of electrically conductive networks. Among the different carbon nano particles, carbon nanofibers, compared to CNTs, possess higher compatibility with the host matrix since their walls are subjected to smaller van der Waals forces thus ensuring better dispersion for longer periods of time [5]. Although there are a large numbers of epoxy resins available, the tetrafunctional epoxy resin, tetraglycidyl methylene dianiline (TGMDA), cured with the aromatic diamine 4,4'-diaminodiphenylsulfone (DDS) is extensively adopted for manufacturing of carbon fiber-reinforced composites (CFRC). This matrix is particularly suited for weight-sensitive applications, such as aircraft vehicles, allowing to achieve fuel/energy minimization and to accomplish sustainability requirements [6]. In the present work, a morphological analysis and an extensive electrical characterization both in DC and AC domain is carried out on samples of TGMDA reinforced with different amount of CNFs up to $2 \mathrm{wt} . \%$. More in details, after investigating the dispersion state of the nanofillers inside the polymeric matrix by means of scanning electron microscopy, the electrical percolation threshold (EPT), the DC volume conductivity and the dielectric characteristics of the nanocomposites in the frequency range $100 \mathrm{~Hz}-1 \mathrm{MHz}$ are analyzed and compared with those exhibited by the pure resin. Moreover, tunneling AFM (TUNA), capable to detect ultra-low currents ranging from $80 \mathrm{fA}$ to $120 \mathrm{pA}$, is adopted as innovative tool for correlating the local topography, in terms of conductive pathways and interconnections in $\mathrm{CNF} /$ resins, with the results concerning the electrical properties.

The interesting electrical properties shown by the CNF/epoxy nanocomposites open the way for developing tailored structures able to meet the demanding industrial requirements in the aeronautic field both as structural materials and for electromagnetic (EM) applications such as coatings, EM shields and filters or radar absorber materials (RAMs).

\section{Experimental section}

\subsection{Materials and Methods}

The epoxy matrix was obtained by mixing TGMDA with BDE monomer at a concentration of $80 \%: 20 \%$ (by weight) epoxide to flexibilizer. Epoxy blend and DDS (at a stoichiometric concentration with respect to the epoxy rings) were mixed at $120{ }^{\circ} \mathrm{C}$ and the carbon nanofibers were incorporated into the matrix by ultrasonication for 20' (Hielsher model UP200S-24kHz high power ultrasonic probe). CNFs, in the form of powders, were produced at Applied Sciences Inc. and belong to the Pyrograf III family. In particular, the as-received CNFs are subjected to a thermal treatment at $2500^{\circ} \mathrm{C}$ since it was found that such a cure is effective in improving both mechanical and electrical properties [7,8]. Different weight percentages $(0.05,0.32,0.64,0.80,1.00,1.30$ and 2.00$)$ of CNFs were dispersed within the epoxy resin. All the obtained mixtures were cured by a two-stage cycle: a first isothermal stage was carried out at $125^{\circ} \mathrm{C}$ for $1 \mathrm{~h}$ followed by a second isothermal stage at higher temperatures up to $200^{\circ} \mathrm{C}$ for $3 \mathrm{~h}$.

Morphological information are achieved by Scanning Electron Microscope-SEM (mod. LEO 1525, Carl Zeiss SMT AG, Oberkochen, Germany) performed directly on the nanofiller and on epoxy/1.3\%CNF nanocomposite, appropriately cut and etched according to a wellestablished procedure already described in previous papers [5]. 
The conductivity mapping at nanoscale level of the nanofilled epoxy samples was performed by TUNA-AFM. The overall setup employs a conductive AFM probe, an external voltage source needed to provide a potential difference between the tip and the sample holder and a current to voltage converter (trans-impedance amplifier, TIA) used to convert the (analogical) current signal into voltages in digital form that can be read and processed by a PC.

In particular, in our experiments TUNA runs with a cantilever holder and an epoxy sample filled with conductive CNFs, electrically connected to an external voltage source. Atomic force microscope (AFM) images were captured at ambient conditons (30\%-40\% humidity) with a Dimension 3100 coupled with a Bruker NanoScope V multimode AFM (Digital Instruments, Santa Barbara, CA) controller operating in tunneling current mode (TUNAAFM), using microfabricated silicon tips/cantilevers. The TUNA-AFM measurements were carried out through platinum-coated probes with nominal spring constants of $35 \mathrm{~N} \mathrm{~m}^{-1}$ and electrically conductive tip of $20 \mathrm{~nm}$. TUNA -AFM operates in contact mode. In feedback mode, the output signal is the DC bias, adjusted to maintain the electric current setpoint. The following values of the TUNA control parameters are adopted: DC sample bias ranged from $1 \mathrm{~V}$ to $2 \mathrm{~V}$, current sensitivity was $1 \mathrm{nA} / \mathrm{V}$, current range was $200 \mathrm{nA}$, samples/lines determining the number of data points or pixels along the $\mathrm{X}$ and $\mathrm{Y}$ axis are 256, scan rate that controls the rate at which the cantilever scans across the sample area was $0.9-1.5 \mathrm{~Hz} \mathrm{~s}^{-1}$. In order to obtain repeatable results, several regions of the specimens were scanned. The images were analyzed using the Bruker software Nanoscope Analysis 1.80 (Build R1.126200). Regarding the electrical characterization, the samples were coated by using a silver paint (RS 186-3600,) with a thickness of about $50 \mu \mathrm{m}$ and a surface resistivity of 0.001 $\Omega \cdot \mathrm{cm}$ (when fully hardened) in order to reduce possible surface roughness and to ensure a good electrical contact with the measuring electrodes. The measurement apparatus for DC characterization of samples above the electrical percolation threshold (i.e. EPT) is composed by a multimeter Keithley $6517 \mathrm{~A}$ operating as voltage generator $(\max \pm 1000 \mathrm{~V})$ and voltmeter $(\max \pm 200 \mathrm{~V})$ and an ammeter HP34401A (min current $0.1 \mu \mathrm{A}$ ). For below the percolation threshold the system is composed exclusively by a multimeter Keithley 6517A working as voltage generator and pico-ammeter (min current $0.1 \mathrm{fA}$ ). The AC properties are measured in the frequency range [0.1-1000] $\mathrm{kHz}$ by using a Quadtec7600 dielectric analyzer and by applying a sinusoidal stimulus of amplitude $0.1 \mathrm{~V}$ or $1 \mathrm{~V}$ for specimens above or below the EPT respectively.

\section{Results and discussions}

\subsection{Morphological Analysis}

In order to analyze the dispersion state of the nanofillers inside the polymeric matrix, the epoxy nanocomposites were investigated by means of SEM analysis carried out. Etched samples are adopted to remove the resin surrounding the nanofibers, leaving them bare.

Fig.1 (on the left) shows SEM image of the fracture surface of the Epoxy $/ 1.3 \%$ CNF sample. A homogeneous structure is achieved since the CNFs, characterized by straight walls (Fig.1, right) are uniformly distributed inside the resin. The heat treatment applied to the filler has prevented agglomeration phenomena encountered with composites obtained by using the asreceived fibers [5].

Moreover, it is worth noting that, in the dark zone of the SEM image, the etching procedure allows to highlight the presence of carbon nanofibers arranged as a continuous network in the matrix thus confirming the interesting electrical behavior revealed by the experimental characterization discussed in the next section. 


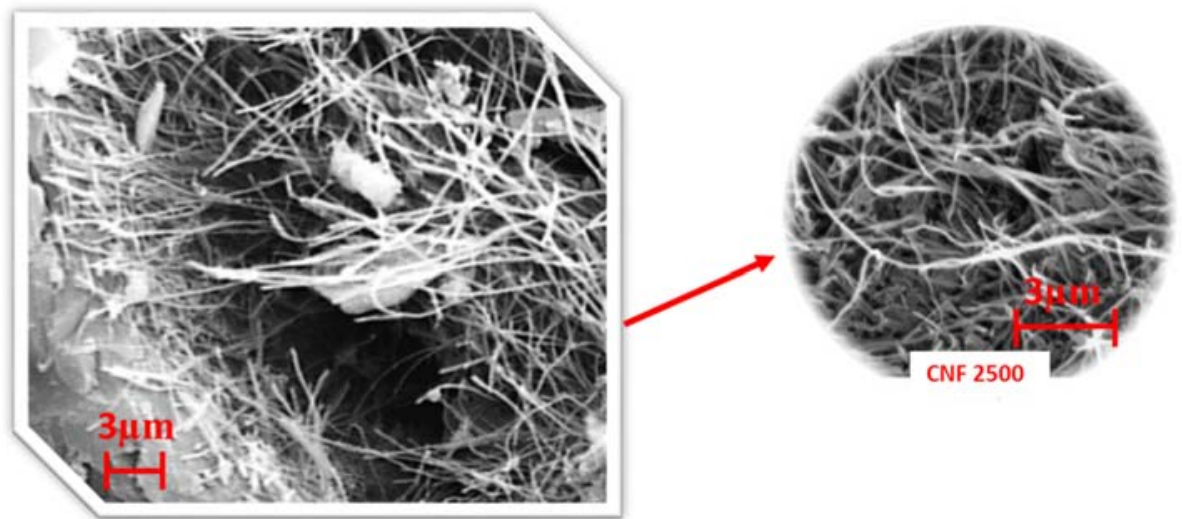

Fig. 1. SEM images of a fracture surface of Epoxy $/ 1.3 \%$ CNF (left) and of the adopted carbon nanofibers, CNF2500 (right).

\subsection{Electrical properties supported by TUNA-AFM investigation}

\subsubsection{DC investigation}

Nanocomposites with varying weight percentage of fillers (up to $2 \mathrm{wt} \%$ ) were prepared and subsequently electrically characterized. In Figure 2 the variation of the bulk conductivity of the composites is reported as a function of increasing filler content.

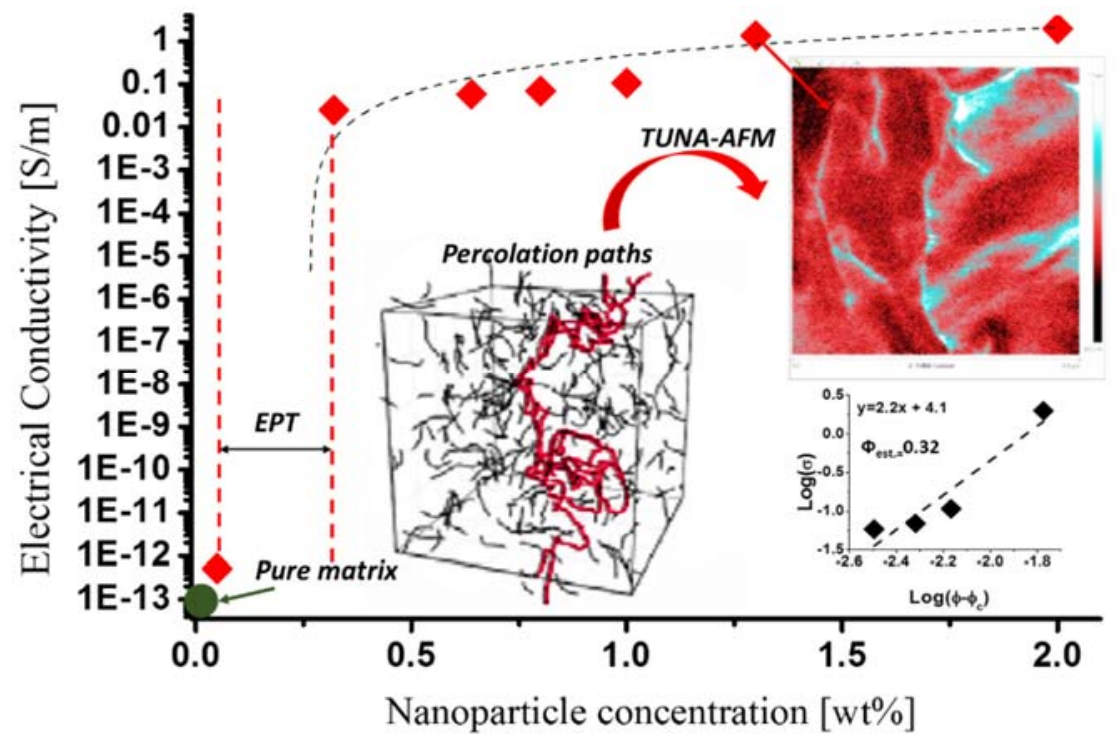

Fig. 2. DC Volume electrical conductivity of nanocomposites as a function of the fillers concentrations (wt\%). Schematic of percolation paths, TUNA-AFM image and log-log plot of the electrical conductivity as function of the filler amount for estimating of the characteristic parameters of the percolation law are shown in the insets.

As shown, the unfilled resin (green circle marker) is an insulating material since its electrical conductivity is of the order of $\mathrm{pS} / \mathrm{m}$. An abrupt increase of the volume conductivity, namely several orders of magnitude with respect to those of insulating compositions, was observed as the electrical percolation threshold $(E P T)$, i.e. the minimum amount of conductive filler 
needed to form at least one continuous electrical path (see inset of Fig.2) within the polymeric resin, is achieved. The experimental results concerning the electrical conductivity provide information about this value that falls in the narrow interval [0.05-0.32] wt.\%. After that, due to an interconnected morphological network established among neighbouring nanoparticles that provides a continuous electrically pathway for the electrons (see, inset of Fig.2, TUNA-AFM image), the conductivity of the composites increases significantly following a trend described by a power-law of the form:

$$
\sigma=\sigma_{0}\left(\phi-\phi_{c}\right)^{t}
$$

where $\sigma_{0}$ is the intrinsic conductivity of the filler, $\phi_{c}$ is the electrical percolation threshold (i.e. EPT) and $t$ is a critical exponent depending on the dimensionality of the percolating structure $[9,10]$.

A significant value of $2 \mathrm{~S} / \mathrm{m}$ is achieved for the conductivity of the nanocomposite at the highest filler loading (i.e. $2 \mathrm{wt} \%$ ).

Moreover, form the graph presenting the log-log plot of the electrical conductivity as function of the filler amount (inset in Fig.2), it is possible to estimate the characteristic parameters of eq.1. A value of 2.2 and $0.32 \mathrm{wt} . \%$ is found for $t$ and for $\phi_{c}$, respectively. More in details, the value for the critical exponent $t$ coincides with the slope of the fitting curve of the experimental data, whereas the value of the estimated electrical percolation threshold (i.e. $\phi_{c .}$ ) acts as a fit parameter to achieve the maximum for the regression coefficient of the estimated curve (i.e. $\mathrm{R}^{2}$ close to 1 ). Such estimated values agree respectively, with typical values reported in literature [11] and with the percolation threshold detected experimentally. As schematized in Fig.3 (left), the electrical conductivity in nanocomposites materials can be interpreted in terms of electron tunneling according to which the electrons jump from a conductive fiber to the closest one over a small distance of the order of a few nanometers [12]. This electrical contribution can be evaluated by means of a suitable resistance, i.e. $R_{\text {tunnel, }}$, as per the following expression:

$$
R_{\text {tunnel }}=\frac{h^{2} d}{A e^{2} \sqrt{2 m \lambda}} \exp ^{\left(\frac{4 \pi d}{h} \sqrt{2 m \lambda}\right)}
$$

where $h$ is the Plank's constant, $A$ and $d$ are the cross-sectional area and the distance between the filler (coincident with the thickness of the insulating resin wrapped around the particles) respectively, $e$ is the electron charge, $m$ is the mass of electron and $\lambda$ is the height of barrier, typically of few eV.

A method reported in literature [13-15] to verify that the electron tunneling is effectively the principal electrical transport mechanism in such nanofilled structures is based on the occurrence, for concentrations ( $\phi$ ) above the EPT, of a linear relation (see Fig. 3, left) between the electrical conductivity (expressed in natural logarithmic scale) and $\phi^{-1 / 3}$, i.e.:

$$
\ln (\sigma) \propto \phi^{-1 / 3}
$$

The straight line is a fit curve of the experimental data (markers): the value of $\mathrm{R}^{2}$ close to 1 confirms that the electrical conduction network in nanocomposites materials is mainly achieved through the quantum tunneling effect. 

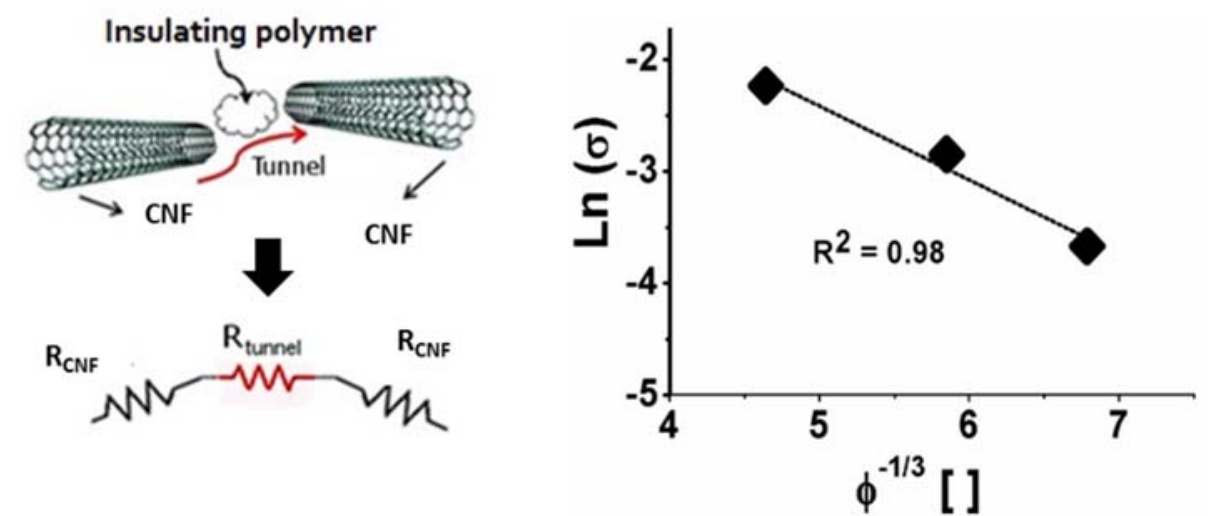

Fig. 3. Schematic of tunneling effect and its electrical representation (left). Plot of the natural logarithm of DC conductivity for sample above the EPT vs. $\phi^{-1 / 3}$ for the validation (right) of the electron tunnelling conduction mode.

\subsubsection{AC investigation}

New advanced materials like nanocomposites whose properties can be modified by varying the amount of fillers dispersed within the host matrix are promising candidates for electromagnetic (EM) applications such as shields, filters and radar absorber materials (RAMs). In order to evaluate their effectiveness for such purposes, the experimental investigation of the AC electrical properties is highly recommended.

Fig. 4 shows the AC electrical conductivity (a) and real part of dielectric permittivity (b) of the nanocomposites evaluated in the frequency range from $100 \mathrm{~Hz}$ up to $1 \mathrm{MHz}$. As it concerns the $\mathrm{AC}$ conductivity, it is worth noting as, at low frequency (i.e. $100 \mathrm{~Hz}$ ) and for each specific nanocomposite system, its value agrees with that measured in DC. In particular, for composites below the percolation threshold, the electrical conductivity presents a strong frequency dependent behaviour similar to that of dispersive (i-e lossy dielectrics) materials. In fact, the conductivity rises gradually with increasing frequency due to the increasing contribution of the frequency dependent displacement current associated to the capacitive effect of materials. On the contrary, composites above the EPT show a frequency independent conductivity, at least in the investigated frequency range.

Particular emphasis shall be given, especially as it concerns the design for electromagnetic purposes, to the complex dielectric permittivity:

$$
\varepsilon=\varepsilon^{\prime}+i \varepsilon^{\prime \prime}
$$

where $\varepsilon^{\prime}$ and $\varepsilon^{\prime \prime}$ are the real and imaginary parts, respectively. More in details, the real part of the dielectric permittivity is associated to the displacement current and it is mainly affected by polarization phenomena within the material, whereas the imaginary part of the permittivity takes into account the losses inside the material. Recently, epoxy based systems are being increasingly investigated for their dielectric properties, since the introduction of fillers demonstrate several enhancement in their properties when compared to that obtained for unfilled epoxy systems. For some interesting electromagnetic applications is required to improve the relative permittivity of such materials. So the dispersion within the epoxy matrix of electrically conductive particles (such as CNFs) with sufficient quantity allows to reach high dielectric values without sacrificing typical features of the host matrix like high breakdown strength and other interesting mechanical, thermal and chemical properties.

Fig. $4 \mathrm{c}$ reports the real part of the complex permittivity as function of the frequency for fillers content up to a total of $2 \mathrm{wt} \%$. As expected, the results show that dielectric permittivity (i.e. $\left.\varepsilon^{\prime}\right)$ increases with respect to that of neat resin with the addition of filler, most likely due to 
the increase in polarization of the matrix/filler interface and/or the interfacial polarization attributable to the conductive particles [16]. A careful analysis of the TUNA current profile (Fig.4 c) of the T20BD $+1.3 \%$ CNF2500 sample confirms the overall macroscopic conductive behaviour (1.37 S/m measured in DC) of the $\mathrm{CNF} /$ resins since currents ranging from $122.8 \mathrm{fA}$ to $1.1 \mathrm{pA}$ were detected at nanoscale level. In fact, the possibility to reveal such low currents (ranging from fA to $\mathrm{pA}$ ) is due to the effective conductive paths established within the matrix, as emerges by the strong colors contrast of the TUNA current micrograph.

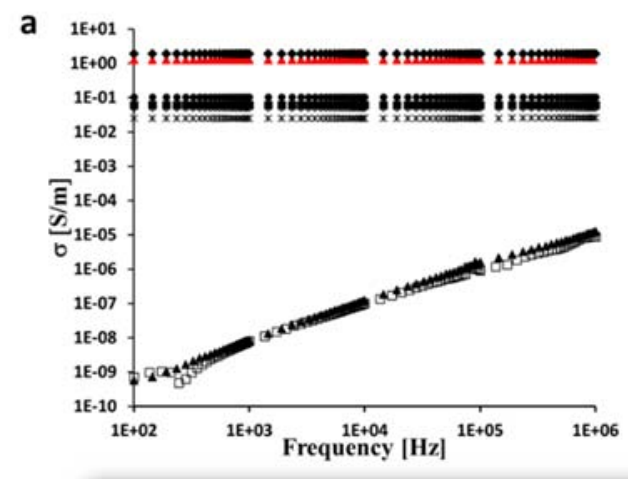

c)

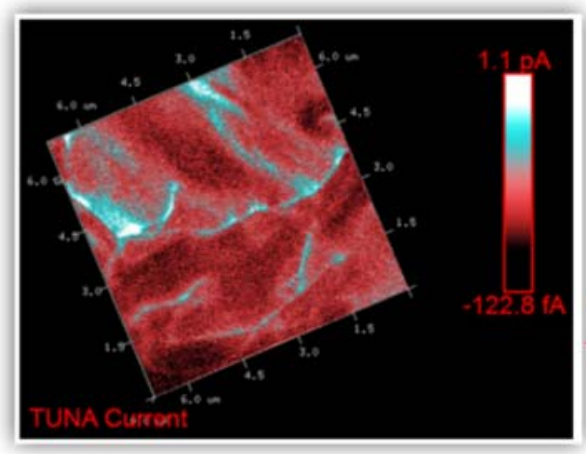

b)

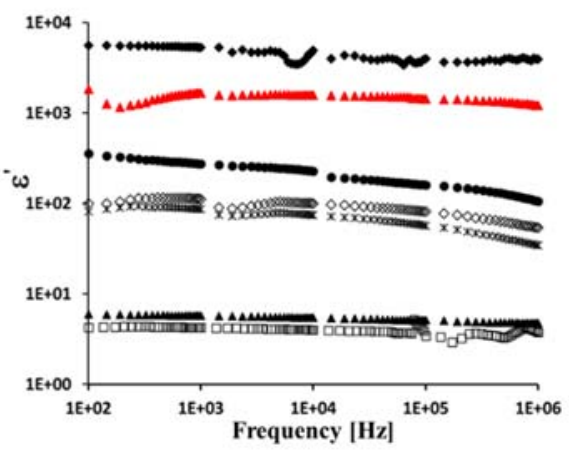

\section{Common Legend}

$\triangle \mathrm{T} 20 \mathrm{BD}$ - Pure Resin

$\square T 20 B D+0.05 \%$ CNF2500

* T20BD + 0.32\% CNF2500

$\diamond \mathrm{T} 20 \mathrm{BD}+0.64 \%$ CNF2500

- T2OBD + 0.8\% CNF2500

- T2OBD + 1\% CNF2500

$\triangle \mathrm{T} 20 \mathrm{BD}+1.3 \%$ CNF2500

- T2OBD + $2 \%$ CNF2500

Fig. 4. Frequency dependency of electrical conductivity (a) and of the real part of complex permittivity (b). TUNA-AFM micrographs $(6.9 \mu \mathrm{m} \times 6.9 \mu \mathrm{m})$ of the fracture surface of the T20BD+1.3\%CNF2500 sample - tuna current image (c).

\section{Conclusions}

An extensive DC and AC electrical characterization on CNF/epoxy nanocomposites has been carried out on a structural resin suitable for aeronautical applications filled with different amounts of carbon nanofibers. It is found that EPT falls in the narrow range $[0.05 \div 0.32] \mathrm{wt} \%$ whereas a remarkable value of electrical conductivity $(2 \mathrm{~S} / \mathrm{m})$ is achieved with a low filler amount ( $2 \mathrm{wt} . \%)$. Moreover, the introduction of small amount of nanofillers increases the relative permittivity (i.e. $\varepsilon$ ') of the resulting materials without sacrificing the dielectric strength and other interesting mechanical, thermal and chemical properties of the host matrix, thus making them suitable for EM applications. TUNA measurements confirm the creation of percolation path in the nanocomposites above the EPT. Further work is underway to correlate TUNA measurements and detected currents with morphological model of the nanocomposites in order to derive information on the conduction mechanisms. 


\section{Acknowledgments}

The research leading to these results has received funding from the European Union's Horizon 2020 research and innovation programme under grant agreement No 760940 MASTRO.

\section{References}

1. L. Guadagno, U. Vietri, M. Raimondo, L. Vertuccio, G. Barra, B. De Vivo, P. Lamberti, G. Spinelli, V. Tucci, F. De Nicola, R. Volponi, S. Russo, Compos. B Eng. 80, pp. 7-14.

2. C. Yang, Y.Lin, C.W.Nan, Carbon 47, pp.1096-1101, (2009).

3. J. Gou, Y. Tang, F. Liang, Z. Zhao, D. Firsich, J. Fielding, Compos B Eng 41, pp. 192198 (2010).

4. L. Guadagno, M. Raimondo, U. Vietri, L. Vertuccio, G. Barra, B. De Vivo, P. Lamberti, G. Spinelli, V. Tucci, R. Volponi, G. Cosentino, F. De Nicola, RSC Adv 5, pp. 60336042 (2015).

5. L. Guadagno L, M. Raimondo, V. Vittoria, L. Vertuccio, L. Khalid, B. De Vivo, P. Lamberti, G. Spinelli, V. Tucci. Nanotechnology. 24, pp. 305704-10, (2013).

6. L. Guadagno, C. Naddeo, M. Raimondo, G. Barra, L. Vertuccio, S. Russo, K. Lafdi, V. Tucci, G. Spinelli, P. Lamberti, Nanotechnology 28, 094001 (2017).

7. B. De Vivo, P. Lamberti, G. Spinelli, V. Tucci, L. Guadagno, M. Raimondo, J. Appl. Phys. 118, 064302 (2015).

8. M. Raimondo, L. Guadagno, V. Speranza, L. Bonnaud, P. Dubois, K. Lafdi, Compos B Eng, 140, pp. 44-56 (2018).

9. L. Guadagno, M. Raimondo, L. Vertuccio, C. Naddeo, G. Barra, P. Longo, P. Lamberti, G. Spinelli, M.R. Nobile. Compos. B Eng. 147, pp. 12-21, (2018).

10. L. Vertuccio, L. Guadagno, G. Spinelli, S. Russo, G. Iannuzzo. Composites Part B 129, pp.1-10, (2017).

11. C.-W. Nan, Y. Shen, Jing Ma, Annu . Rev. Mater. Res. 40, pp. 131-151 (2010).

12. Chunyu Li, Erik T. Thostenson, Tsu-Wei Chou, Appl. Phys. Lett. 91, 223114 (2007).

13. B. E. Kilbride, J. Coleman, J. Fraysse, P. Fournet, M. Cadek, A. Drury, S. Hutzler, S. Roth S and W. Blau. J. Appl. Phys. 92, pp. 4024-30, (2002).

14. A. Mdarhri, F. Carmona, C. Brosseau C and P. Delhaes. J. Appl. Phys. 103, 054303, (2008).

15. M. T. Connor, S. Roy, T. A. Ezquerra and F. J. B. Calleja. Phys. Rev. B 57, pp.22862294, (1998).

16. V. Panwar, J. O. Park, S. H. Park, S. Kumar,R. M. Mehra. J. Appl. Polym. Sci., 115, pp. 1306-1314, (2010). 